\title{
$\mathrm{MAPbI}_{3}$ 구조를 기반으로 한 하이브리드 유무기 할라이드 페로브스카이트 구조의 합리적인 DFT 계산 방법 연구 \\ 이진웅 ${ }^{1}$ 박운배 ${ }^{2, *}$ \\ 1세종대학교 나노신소재공학과 \\ 2순천대학교 인쇄전자공학과
}

\section{A Reasonable DFT Calculation Method for Hybrid Organic-Inorganic Halide Perovskites}

\author{
Jin-Woong Lee ${ }^{1}$ and Woon Bae Park,* \\ ${ }^{1}$ Faculty of Nanotechnology and Advanced Materials Engineering, Sejong University, Seoul 05006, Republic of Korea \\ ${ }^{2}$ Department of Printed Electronics Engineering, Sunchon National University, Chonnam 57922, Republic of Korea
}

\begin{abstract}
Hybrid Organic-Inorganic Perovskites (HOIP) have received a great deal of attention as a key material for applications like solar cells and light emitting devices because of their many advantages, in spite of their stability and toxicity issues. Attempting to discover and characterize novel HOIPs using just an experimental approach would be prohibitively time-and-cost-consuming. Using theoretical or empirical calculations would greatly help. For these reasons, HOIP has been actively investigated using DFT (Density Functional Theory) calculations, which have significantly reduced research time and cost. However, the input model structure treatment needs to be standardized to avoid unnecessary complications. For this purpose, a sort of optimization of DFT calculation protocols for HOIPs is essential, because DFT calculation results are greatly affected by the input model structure arrangements and exchange-correlation functionals. In this paper, we used DFT to calculate the band gap, formation energy, and effective mass of the well-known cubic perovskite structure, methylammonium lead iodide $\left(\mathrm{CH}_{3} \mathrm{NH}_{3} \mathrm{PbI}_{3}: \mathrm{MAPbI}_{3}\right)$ with and without the van der Waals function and SOC (Spin Orbit Coupling) and various geometrical molecule arrangements in the structure. In particular, the initial orientation of the 'A' site molecule in the input model structure was intensively investigated in terms of band gap, formation energy and effective mass. It was found that the relaxation-induced final structure was greatly influenced by the initial orientation of the molecule and thereby significantly affected the DFT-calculated result.
\end{abstract}

(Received February 10, 2021; Accepted February 18, 2021)

Keywords: hybrid organic-inorganic perovskite, density functional theory, band gap, effective mass, formation energy

\section{1. 서 론}

하이브리드 유무기 할라이드 페로브스카이트 구조(hybrid organic/inorganic perovskite: HOIP)는 높은 광 흡수 계수, 우수한 캐리어 이동도 및 저온, 저가 공정이 가능하다는 장점으로 지난 10 년 동안 태양전지의 광 흡수체로 사용되

- 이진웅: 연구원, 박운배: 교수

*Corresponding Author: Woon Bae Park

[Tel: +82-61-750-5262, E-mail: wbpark@scnu.ac.kr]

Copyright (C) The Korean Institute of Metals and Materials
면서 최근 몇 년 만에 25.5\% [1-4]에 이르는 매우 향상된 전력변환 효율을 보여주고 있다. 또한, 우수한 발광 효율로 발광 다이오드, 레이저, 광 검출기 등의 다양분야로도 응용 이 되고 있다. 이러한 $\mathrm{HOIP}$ 구조는 $\mathrm{AxByXz}$ 화학식으로 되어 있으며, 합성 조건 및 $\mathrm{A}$ 자리의 유기물 종류, $\mathrm{B}$ 양 이온, $\mathrm{X}$ 음이온 자리의 원소 종류 및 구조를 이루는 조성 에 따라 structure type이 달라진다. 이러한 조성 및 구조 변화의 용이성으로 특성이 변화하여 많은 연구자들의 주목 을 받고 있으며, 새로운 조성의 연구가 활발히 진행되고 있다 [5-21]. 
많은 $\mathrm{HOIP}$ 구조 중에 3차원 구조를 가지는 $\mathrm{ABX}_{3}$ type 페로브스카이트 구조가 가장 많이 연구되었으며, $\mathrm{ABX}_{3}$ 화 학식에서 $\mathrm{A}$ 양이온 자리에 대표적으로 많이 사용하는 유 기물로는 메틸암모늄(MA: Methylammonium, $\mathrm{CH}_{3} \mathrm{NH}_{3}{ }^{+}$)이 나 포름아미디늄(FA: Formamidinium, $\mathrm{CH}\left(\mathrm{NH}_{2}\right)_{2}{ }^{+}$), 에틸 암모늄(EA: ethylammonium, $\mathrm{CH}_{3} \mathrm{CH}_{2} \mathrm{NH}_{3}{ }^{+}$), 과니디늄 $\left(\mathrm{GA}\right.$ : guanidinium, $\left[\mathrm{C}\left(\mathrm{NH}_{2}\right)_{3}\right]^{+}$) 등이 있으며, $\mathrm{B}$ 양이온 자리에는 납, 주석, 저마늄, $\mathrm{X}$ 음이온 자리에 요오드, 브롬 및 염소와 같은 할로겐 원소가 자리를 차지하거나 공유한 다. A 자리 유기물의 경우 앞서 언급한 유기물을 제외하 고도 수많은 유기물에 대한 연구가 활발히 진행되고 있으 며, B 양이온 및 X 음이온 또한 연구되고 있다. 하지만, 지식과 경험을 기반으로 하는 실험적 접근법에는 많은 경 우의 수를 가지는 HOIP 실험에 한계가 있다. 이러한 실험 적 한계로 비용 및 시간을 절감 할 수 있는 DFT 계산 방법론이 대두되고 있으며 [22-33], 많은 수의 DFT 계산 결과를 가지고 새로운 페로브스카이트 발견을 용이하게 하 는 소재 머신 러닝 전략 [34-37]이 주목을 받고 있다 [38-40]. 하지만, HOIP의 DFT 계산은 몇 가지의 중요한 문제점을 가지고 있으며, 가장 큰 문제점은 결정 구조에서 유기물 분자의 위치를 특정 할 수 없다는 데에 있다. 이러 한 유기물 분자의 경우 일부 구조가 해석되어 있지만, 대 부분은 무작위로 계산 모델을 만들어 DFT 계산을 수행한 다. 이러한 무작위 계산 모델은 DFT 계산의 상이한 결과 로 나오지만, 대부분의 연구자들은 이러한 문제에 대해 깊 게 취급하지 않고 있다. 또 다른 문제점은 DFT 계산의 교환 상관 함수이다. DFT 계산은 교환 상관 함수에 따라 상이한 결과가 나온다. 이 문제점은 잘 알려진 사실이며, 여러 구조를 대상으로 한 많은 연구에서 보고되었다 [4143]. 이러한 맥락에서 재료 특성(전자 및 열역학)을 합리적 으로 계산할 수 있는 계산 접근법이 필수적이다. 본 연구 에서 우리는 비교적 간단한 $\mathrm{MAPbI}_{3} \mathrm{HOIP}$ 구조를 대상으 로 $\mathrm{DFT}$ 계산을 수행하여, 다른 종류의 HOIP 구조에도 적용할 수 있는 합리적인 DFT 계산 접근법을 찾고자 연 구를 수행하였다.

\section{2. 계산 방법}

DFT 계산은 Vienna ab initio simulation package (VASP5.4)[44-48] 프로그램을 사용하여 수행되었으며, 공 통적으로 교환 상관 에너지는 GGA-PBE(generalized gradient approximation parameterized by Perdew, Burke, and Ernzerhof) [44], pseudopotential로 PAW (projector-augmented-wave) potential [49,50], k-mesh 샘 플링에는 Monkhorst-Pack 기법을 채택하였으며, K-mesh는 $2 \times 2 \times 2$ 이다. 또한, 모든 계산에서 cut-off energy는 $400 \mathrm{eV}$, Total energy convergence는 $10^{-4} \mathrm{eV}$ 로 수행되었 으며, 개별 원자에 가해지는 힘을 $0.05 \mathrm{eV} / \AA$ 이하로 최소 화하였다.

\section{3. 결과 및 고찰}

\section{1계산 모델}

합리적인 DFT 계산 접근법을 찾고자 선택한 $\mathrm{MAPbI}_{3}$ HOIP 구조는 지금까지 합성 조건과 환경에 따라 Cubic(Pm-3m), Tetragonal(I4/mcm), Orthorhombic(Pnma) 구조로 알려져 있다 [51]. 본 연구에서 DFT 계산에 사용 된 초기 구조는 $P m-3 m$ 공간 그룹 갖는 $\mathrm{CsPbI}_{3}$ (ICSD_161481) Cubic 구조로 선택하였다. 이러한 $\mathrm{CsPbI}_{3}$ Cubic 구조를 선택한 이유는 치환되는 유기물 분자인 $\mathrm{MA}\left(\mathrm{CH}_{3} \mathrm{NH}_{3}{ }^{+}\right)$분자 때문이다. $\mathrm{MA}\left(\mathrm{CH}_{3} \mathrm{NH}_{3}{ }^{+}\right)$분자는 일 반적인 원소보다 크기 때문에 치환되는 site에 충분한 공간 이 있어야 유리하기 때문이다. 또한, $\mathrm{CsPbI}_{3} \mathrm{Cubic}$ 구조에 $\mathrm{MA}\left(\mathrm{CH}_{3} \mathrm{NH}_{3}{ }^{+}\right)$분자를 치환하면 결정학적으로 symmetry가 깨지고, 구조는 $\operatorname{triclinic}(P 1)$ 이 된다. 이러한 상황에서 $\mathrm{DFT}$ 계산을 수행하면, 구조 이완 과정을 통해 완전히 $\operatorname{symmetry}$ 는 $\operatorname{triclinic}(P 1)$ 이 된다. 따라서 초기 구조는 DFT 계산 수행에 용이 하면서 가장 간단한 구조인 Cubic 구조로 선택하였다. 선택된 $\mathrm{CsPb}_{3}\left(\mathrm{ICSD} \_161481\right)$ Cubic 구조의 $\mathrm{Cs}$ 원소 자리에 서로 다른 기하학적 모양을 갖는 27 개의 $\mathrm{MA}\left(\mathrm{CH}_{3} \mathrm{NH}_{3}{ }^{+}\right)$분자를 치환하여 27개의 계산 모델 을 만들었다. 또한, 단위 셀 상태보다 DFT 계산의 정확성 을 높이기 위해 모든 모델은 2 X 2 X 2 Super cell 상태에 서 계산되었다. 전체적인 계산 모델을 만드는 과정을 그림 1 에 나타내었다.

그림 1(a)를 보면 알 수 있듯이 $\mathrm{MA}\left(\mathrm{CH}_{3} \mathrm{NH}_{3}{ }^{+}\right)$분자의 서로 다른 기하학적 모양은 $\mathrm{MA}$ 분자의 무게 중심을 기준 으로 C-N 본드가 $\mathrm{z}$ 축 위에 있을 때를 기준으로 $\mathrm{x}$ 축, $\mathrm{y}$ 축, $\mathrm{z}$ 축을 각 축 마다 $0 \sim 60$ 도 사이에서 무작위로 설정하 였다. 무작위로 설정한 $\mathrm{MA}\left(\mathrm{CH}_{3} \mathrm{NH}_{3}{ }^{+}\right)$분자는 그림 1(d)에 나타내었으며, 이렇게 생성된 $\mathrm{MA}\left(\mathrm{CH}_{3} \mathrm{NH}_{3}{ }^{+}\right)$분자를 그림 1(b)와 같이 Cs 원소 자리에 치환하였으며, 마지막으로 2 X 2 X 2 Super cell을 생성하였다. 최종적으로 27개의 계 산 모델 구조를 생성하였으며, 4가지(GGA, GGA+SOC, $\mathrm{GGA}+\mathrm{D} 3, \mathrm{GGA}+\mathrm{D} 3+\mathrm{SOC}$ ) 다른 상태의 $\mathrm{DFT}$ 계산을 수 행하여 형성 에너지, 밴드 갭, 유효 질량을 조사하였다. 

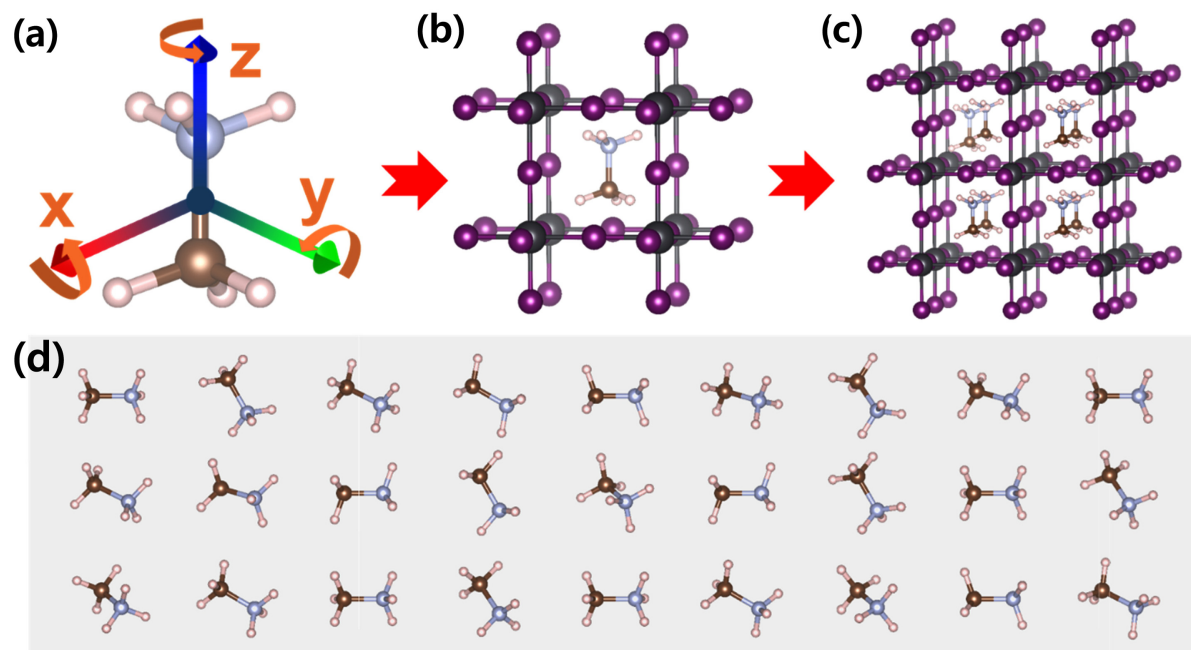

Fig. 1. Schematic represent atom for the rotation of $\mathrm{MA}\left(\mathrm{CH}_{3} \mathrm{NH}_{3}{ }^{+}\right)$molecule. 3 orange arrows represent the rotation of molecule around each $\mathrm{x}, \mathrm{y}, \mathrm{z}$ axis. (b) The representation of substituted $\mathrm{CsPbI}_{3}$ cubic structure with MA. (c) The calculated 2 X 2 X 2 supercell structure of (b). (d) The 27 MAs made from the method represented in (a) with randomly selected degrees.

\section{2 형성 에너지 계산}

모든 소재의 불안정성은 온도, 습도, 열, 빛, 산소 등의 조건에서 야기되며, 이러한 이유로 HOIP 소재 또한 장기 적 안정성에 관한 많은 연구가 진행되었다 [52-59]. HOIP 소재는 습한 환경에 노출되면 급격히 분해된다. HOIP 구 조의 열 안정성은 그 형성 에너지를 통해 결정된다. $\mathrm{MAPbI}_{3}$ 는 열 적으로 불안정하며, $333 \mathrm{~K}$ 이상에서 $\mathrm{MAI}$ $+\mathrm{PbI}_{2}$ 화합물로 분해된다 [60]. 마찬가지로, 본 연구는 $\mathrm{MAPbI}_{3} \rightarrow \mathrm{MAI}+\mathrm{PbI}_{2}$ 형식의 분해를 고려하여 형성 에너 지를 계산하였다. 형성 에너지는 식 (1)과 같이 계산할 수 있다.

$$
\Delta \mathrm{H}=\mathrm{E}\left(\mathrm{MAPbI}_{3}\right)-\left[\mathrm{E}(\mathrm{MAI})+\mathrm{E}\left(\mathrm{PbI}_{2}\right)\right]
$$

우리는 식 (1)을 통해 기하학적으로 서로 다른 27개의 $\mathrm{MAPbI}_{3} \mathrm{HOIP}$ 조조와 4 가지(GGA, GGA+SOC, GGA $+\mathrm{D} 3, \mathrm{GGA}+\mathrm{D} 3+\mathrm{SOC})$ 다른 상태의 $\mathrm{DFT}$ 계산을 수행하여 형성 에너지를 계산 했다.

일반적인 DFT 계산에서 형성 에너지 계산은 음수의 값 이 나오지만 HOIP 구조 같이 특정 분자가 구조에 원소 대신 치환된 특수한 상황에서 형성 에너지 계산은 종종 양 수 값을 가진다. 이러한 계산에서 중요한 점은 비록 양수 로 나올지라도 동일한 조건에서 나온 경향성 있는 결과는 충분한 의미가 있다.

그림 2의 $\mathrm{GGA}, \mathrm{GGA}+\mathrm{SOC}, \mathrm{GGA}+\mathrm{D} 3, \mathrm{GGA}+\mathrm{D} 3+$ $\mathrm{SOC}$ 계산 조건에서 각각의 형성 에너지 분포를 보면 계 산 모델에 따라 $\mathrm{GGA}$ 는 0.341 0.449 eV/f.u., GGA+

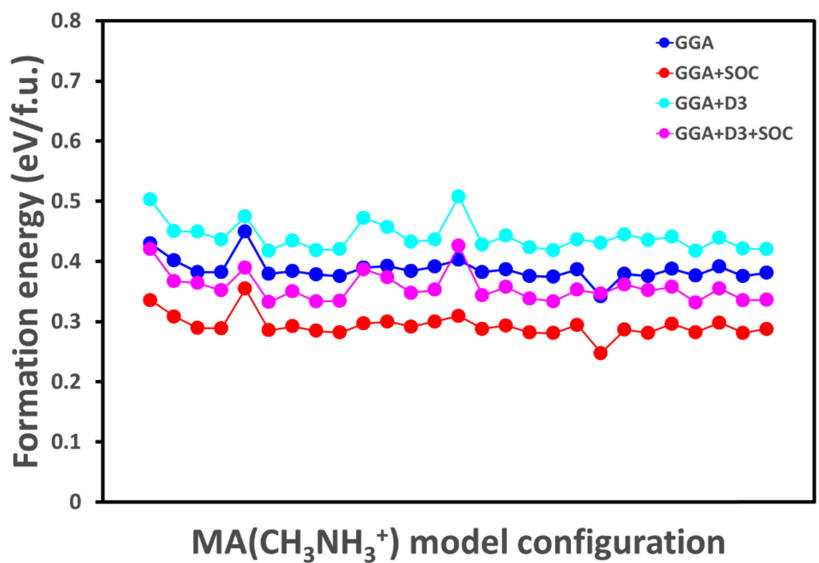

Fig. 2. Results of formation energy calculation using different molecular configurations with four different types of DFT calculations (GGA, GGA+SOC, GGA+D3, GGA+D3+SOC).

$\mathrm{SOC}$ 는 $0.247 \sim 0.354 \mathrm{eV} /$ f.u., GGA+D3는 $0.417 \sim 0.507$ eV/f.u., GGA+D3+SOC는 $0.331 \sim 0.425 \mathrm{eV} /$ f.u. 분포를 보 인다. 각각의 분포 차이는 $0.108,0.107,0.09,0.094$ 로 나왔다. 이러한 형성 에너지 계산 결과를 보면 알 수 있듯 이 계산 모델이 형성 에너지에 많은 영향을 미치는 것을 알 수 있다. 이러한 이유는 $\mathrm{Cs}$ 원소 자리에 $\mathrm{MA}\left(\mathrm{CH}_{3} \mathrm{NH}_{3}{ }^{+}\right)$분 자가 치환되어 구조 이완 과정에서 구조를 심각하게 왜곡 시키기 때문이다. 하지만, 계산 조건에 따라서는 경향성이 일치함을 알 수 있다.

\subsection{Band structure and Effective mass}

$\mathrm{MA}\left(\mathrm{CH}_{3} \mathrm{NH}_{3}{ }^{+}\right)$분자가 $\mathrm{CsPbI}_{3}\left(\mathrm{ABX}_{3}\right)$ Cubic 구조에서 


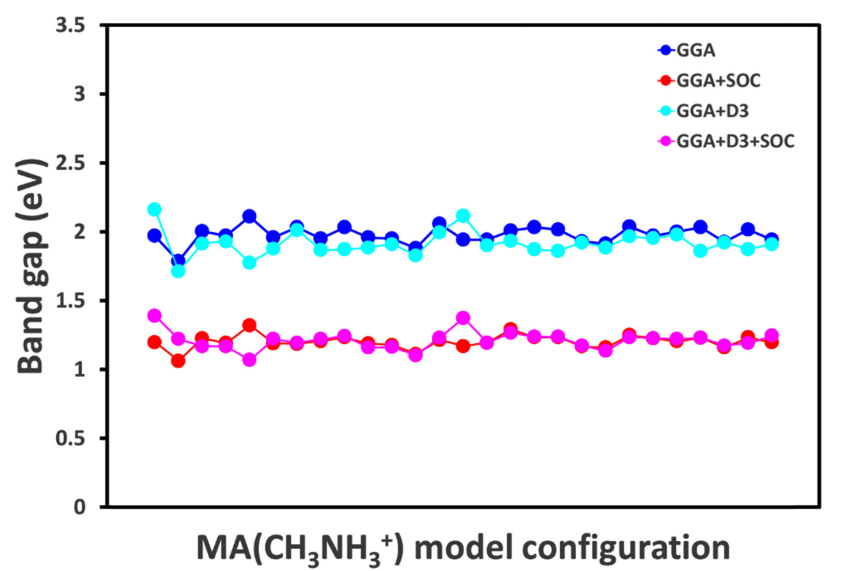

Fig. 3. The band gap of each configurations following four different types of DFT calculations (GGA, GGA+SOC, GGA+D3, GGA+ D3+SOC).

$\mathrm{Cs}$ 원소가 위치한 A site에 치환되면, 엄격한 결정학적 관 점에서 기존의 Cubic 대칭을 잃어버리게 된다. 따라서 모 든 분자 치환 구조는 $P 1$ 대칭을 갖는 것으로 간주하는 것 이 합리적이다. DFT 계산 수행에서 구조적 이완이 제약 없이 완료되었을 때, 이완에 의한 왜곡으로 인해 기존의 대칭은 더욱 흐트러진다. 이러한 상황에서 밴드 구조를 구 성하는 방법에는 두 가지가 있다. 첫 번째는 $P 1$ 대칭의 브릴루앙 영역으로 밴드 구조를 구성하는 것이고, 두 번째 는 원래 구조 대칭의 브릴루앙 영역으로 밴드 구조를 구성 하는 방법이다. 엄격한 결정학적 관점에서는 비록 $P 1$ 대칭 이지만, 기존의 모든 보고에서 밴드 구조 구성을 원래의 대칭성을 사용하였기 때문에 본 연구에서도 원래의 대칭성 을 사용하였다. 또한, $P 1$ 구성과 원래의 대칭에 따른 밴드 구조 구성은 많은 밴드 갭 차이로 이어지지 않지만, 서로 다른 유효 질량 값을 발생시킨다. 밴드 갭 차이보다 유효 질량의 차이가 크게 나는 이유는 특정 $\mathrm{k}$ 지점에서 측정한
유효 질량이 대칭 설정에 영향을 더 많이 받기 때문이다.

그림 3 은 기하학적으로 서로 다른 27 개의 $\mathrm{MAPbI}_{3}$ $\mathrm{HOIP}$ 구조와 4 가지 $(\mathrm{GGA}, \mathrm{GGA}+\mathrm{SOC}, \mathrm{GGA}+\mathrm{D} 3$, $\mathrm{GGA}+\mathrm{D} 3+\mathrm{SOC}$ ) 다른 조건의 $\mathrm{DFT}$ 계산을 수행하여 계산 된 Band Gap 값을 보여준다. 계산 된 결과를 보면 크게 SOC(Spin Obrit Coupling) 상호작용을 포함한 계산과 포 함하지 않는 경우로 나누어지며, 유기물인 $\mathrm{MA}\left(\mathrm{CH}_{3} \mathrm{NH}_{3}{ }^{+}\right)$ 분자에 의해 이완된 구조에 따라 상이한 결과를 나타내었 다. 일반적인 DFT 계산에서 Band gap은 대부분 과소평가 되어 좀더 정확한 계산을 위해 HSE06 계산을 통해 보정 한다 [61-67]. 하지만 $\mathrm{MAPbI}_{3} \mathrm{HOIP}$ 구조에서는 실험 광 학 밴드 갭인 $1.55 \mathrm{eV}$ 보다 높은 과대평가의 결과가 나왔 으며, SOC(Spin Obrit Coupling) 상호작용을 포함한 계산 의 경우에는 과소평가 결과가 나왔다.

작고 균형 잡힌 유효 질량은 태양전지에서 전하 분리, 발광체에서 전하 재조합에 유리하기 때문에 작고 균형 잡 힌 전자 $\left(\mathrm{m}_{\mathrm{e}}{ }^{*}\right)$ 및 홀 $\left(\mathrm{m}_{\mathrm{h}}{ }^{*}\right)$ 를 필요로 한다. 여기서 $\mathrm{m}_{\mathrm{e}}$ *는 $\mathrm{CBM}$ (conduction band minimum) 곡률에서 도출한 유효 질량이며, $\mathrm{m}_{\mathrm{h}}$ *는 $\mathrm{VBM}$ (valence band maximum) 곡률에 서 도출한 유효 질량이다. 그림 4는 기하학적으로 서로 다른 27 개의 $\mathrm{MAPbI}_{3} \mathrm{HOIP}$ 구조와 4가지(GGA, GGA+SOC, $\mathrm{GGA}+\mathrm{D} 3, \mathrm{GGA}+\mathrm{D} 3+\mathrm{SOC}$ ) 다른 상태의 $\mathrm{DFT}$ 계산에서 도출한 유효 질량을 나타낸다. 그림 4의 (a) $\mathrm{m}_{\mathrm{e}}^{*}$ 와 (b) $\mathrm{m}_{\mathrm{h}}$ *를 보면 앞서 언급한 형성에너지나 Band gap 보다 매 우 많은 차이를 나타내는 것을 알 수 있다. $\mathrm{m}_{\mathrm{e}}$ * 경우 특 히 $\mathrm{GGA}+\mathrm{SOC}$ 계산에서 매우 큰 값의 유효 질량이 나왔 으며, 그 차이는 매우 심각하게 나왔다. $\mathrm{m}_{\mathrm{h}}$ * 경우에서는 $\mathrm{GGA}+\mathrm{D} 3+\mathrm{SOC}$ 계산에서 27 개의 계산 모델 중에 12 개의 계산 모델에서 매우 작은 값의 유효 질량 값을 보였다. 유 효 질량 계산을 전체적으로 보면 DFT 계산 방법에서도 차이가 일어나지만, 그 보다 계산 모델에 따른 차이가 매
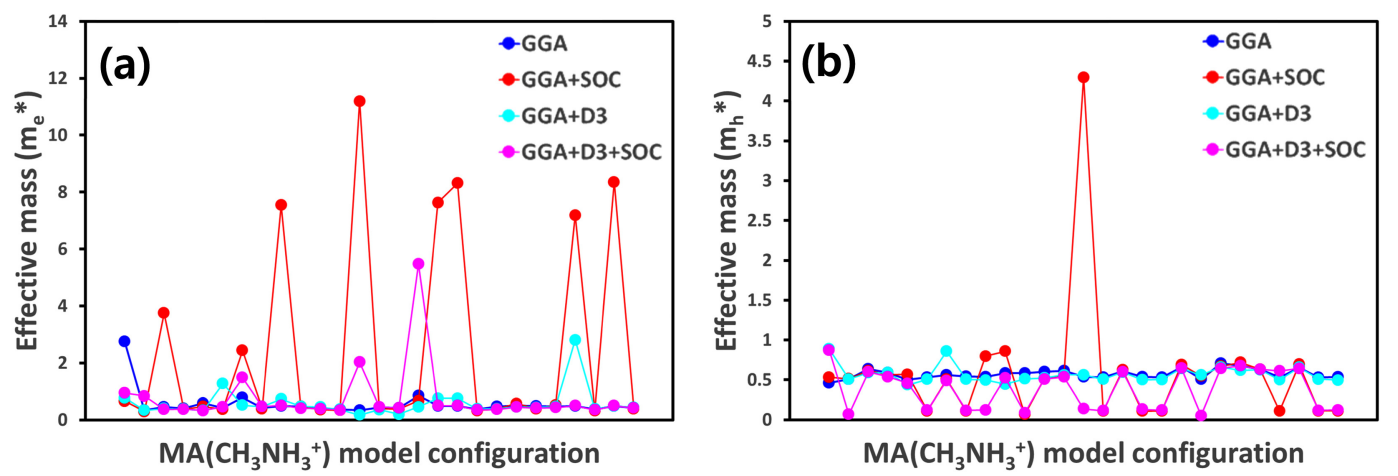

Fig. 4. The effective mass calculation resulted from 4 different DFT calculations (GGA, GGA+SOC, GGA+D3, GGA+D3+SOC). (a) The plot of effective mass of electron calculated from CBM (conduction band minimum) curvature. (b) The plot of effective mass of hole calculated from VBM (valence band minimum) curvature. 
우 큰 것을 알 수 있다. 이러한 이유는 구조 이완 과정에 서 유기물인 $\mathrm{MA}\left(\mathrm{CH}_{3} \mathrm{NH}_{3}{ }^{+}\right)$분자가 구조를 심각하게 왜곡 시켜 $\mathrm{CBM} / \mathrm{VBM}$ 의 곡률을 변화 시키기 때문이다. HOIP 구조의 유효 질량을 계산한 논문들을 보면 대부분 $\mathrm{SOC}$ 기능을 포함하여 계산하였다. 본 연구 결과에서 보듯이 일 부에서 매우 작은 값으로 나오지만 계산 모델에 따라 그 차이가 심각하게 일어나는 것을 보았을 때 가장 중요한 것 은 계산 모델임을 확인하였다.

\section{4. 결 론}

본 연구는 HOIP 구조의 합리적인 DFT 계산 방법을 찾 고자 수행되었으며, HOIP 구조의 가장 큰 문제점인 유기 물 분자의 위치를 특정 할 수 없다는 데에서 시작하였다. 우리는 HOIP 3 차원 구조 중 가장 간단한 $\mathrm{MAPbI}_{3} \mathrm{HOIP}$ 구조를 대상으로 기하학적으로 서로 다른 27 개의 계산 모 델을 $\mathrm{GGA}, \mathrm{GGA}+\mathrm{SOC}, \mathrm{GGA}+\mathrm{D} 3, \mathrm{GGA}+\mathrm{D} 3+\mathrm{SOC}$ 4가 지 조건으로 $\mathrm{DFT}$ 계산을 수행하여 형성 에너지, Band $\mathrm{gap}$, 유효 질량 $\left(\mathrm{m}_{\mathrm{e}}{ }^{*}, \mathrm{~m}_{\mathrm{h}}{ }^{*}\right)$ 을 계산하여 비교하였다. 그 결 과 모든 결과에서 계산 모델에 따른 차이점을 보였으며, 계산 조건에 따라서는 비슷한 경향이 보임을 확인하였다. 하지만, 유효 질량의 경우에는 차이가 심각하게 나타났다. 이러한 모든 결과는 서로 다른 27 개 계산 모델의 구조 이 완 과정에서 일어났으며, 그 이유는 유기물인 $\mathrm{MA}$ $\left(\mathrm{CH}_{3} \mathrm{NH}_{3}{ }^{+}\right)$분자가 구조를 심각하게 왜곡 시켰기 때문이다. 따라서 HOIP 구조의 DFT 계산에서 합리적인 형성 에너 지, Band gap, 유효 질량의 계산을 위해서는 동일한 계산 조건으로 수행되어야 하며, 서로 다른 여러 개의 모델에 대해 DFT 계산을 할 필요성이 있다.

\section{감사의 글}

이 성과는 정부(과학기술정보통신부)의 재원으로 한국연 구재단(NRF-2014R1A6A1030419, 2018R1C1B6006943)과 2020년 순천대학교 학술연구비(2020-0198) 공모과제로 연 구되었음.

\section{REFERENCES}

1. P. Basera, M. Kumar, S. Saini, and S. Bhattacharya, Phys. Rev. B 101, 054108 (2020).

2. N. G. Park, M. Grätzel, T. Miyasaka, K. Zhu, and K. Emery, Nat. Energy 1, 16152 (2016).

3. W. S. Yang, B. Park, E. H. Jung, and N. J. Jeon, Science
356, 1376 (2017).

4. National Renewable Energy Laboratory, Best Research-Cell Efficiencies, https://www.nrel.gov/pv/cell-efficiency.html (2021).

5. A. Kojima, K. Teshima, Y. Shirai, and T. Miyasaka, J. Am. Chem. Soc. 17, 6050 (2009).

6. M. M. Lee, J. Teuscher, T. Miyasaka, T. N. Murakami, and H. J. Snaith, Science 338, 643 (2012).

7. S. D. Stranks, G. E. Eperon, G. Grancini, C. Menelaou, M. J. P. Alcocer, T. Leijtens, L. M. Herz, A. Petrozza, and H. J. Snaith, Science 342, 341 (2013).

8. M. Liu, M. B. Johnston, and H. J. Snaith, Nature 501, 395 (2013).

9. Q. A. Akkerman, G. Rainò, M. V. Kovalenko, and L. Manna, Nat. Mater. 17, 394 (2018).

10. D.-Y. Son, S.-G. Kim, J.-Y. Seo, S.-H. Lee, H. Shin, D. Lee, and N.-G. Park, J. Am. Chem. Soc. 140, 1358 (2018).

11. A. K. Jena, A. Kulkarni, and T. Miyasaka, Chem. Rev. 119, 3036 (2019).

12. L. Mao, C. C. Stoumpos, and M. G. Kanatzidis, J. Am. Chem. Soc. 141, 1171 (2019).

13. C. Shi, L. Ye, Z.-X. Gong, J.-J. Ma, Q.-W. Wang, J.-Y. Jiang, M.-M. Hua, C. F. Wang, H. Yu, Y. Zhang, and H.-Y. Ye, J. Am. Chem. Soc. 142, 545 (2020).

14. J. Xu, X. Li, J. Xiong, C. Yuan, S. Semin, T. Rasing, and X.H. Bu, Adv. Mater. 32, 1806736 (2019).

15. T. Nakajima and K. Sawada, J. Phys. Chem. Lett. 8, 4826 (2017).

16. Y. Li and K. Yang, Energy Environ. Sci. 12, 2233 (2019).

17. S. Lu, Q. Zhou, Y. Ouyang, Y. Guo, Q. Li, and J. Wang, Nat. Commun. 9, 3405 (2018).

18. Z. Li, Q. Xu, Q. Sun, Z. Hou, and W.-J. Yin, Adv. Funct. Mater. 29, 1807280 (2019).

19. J. Schmidt, J. Shi, P. Borlido, L. Chen, S. Botti, and M. A. L. Marques, Chem. Mater. 29, 5090 (2017).

20. J. Jeong, D. Song, J. Choe, C.-H. Chung, and K.-H. Hong, Korean J. Met. Mater. 57, 535 (2019).

21. B. T. Tan, J. Zhang, K. V. Sopiha, and P. Wu, Met. Mater. Int. 25, 869 (2019).

22. M. Kim, S. P. Singh, S. Shim, W. B. Park, and K.-S. Sohn, Chem. Mater. 32, 6697 (2020).

23. W. B. Park, M. G. T. Nathan, S. C. Han, J.-W. Lee, K.-S. Sohn, and M. Pyo, RSC Adv. 10, 43273 (2020).

24. S. C. Han, W. B. Park, K.-S. Sohn, and M. Pyo, Inorg. Chem. Front. 7, 2023 (2020).

25. J. Hong, J. Lee, Y.-W. Lee, W. B. Park, D. Ahn, J. B. Park, 
S. Pak, J. Baik, S. M. Morris, S. Cha, K.-S. Sohn, and J. I. Sohn, J. Power Sources 444, 227301 (2019).

26. S. J. Richard Prabakar, A. B. Ikhe, W. B. Park, K.-C. Chung, H. Park, K.-J. Kim, D. Ahn, J. S. Kwak, K.-S. Sohn, and M. Pyo, Adv. Sci. 6, 1902129 (2019).

27. S. C. Han, W. B. Park, K.-S. Sohn, and M. Pyo, J. Solid State Electr. 23, 3135 (2019).

28. N. Naveen, W. B. Park, S. P. Singh, S. C. Han, D. Ahn, K.S. Sohn, and M. Pyo, Small 14, 1803495 (2018).

29. W. B. Park, S. C. Han, C. Park, S. U. Hong, U. Han, S. P. Singh, Y. H. Jung, D. Ahn, K.-S. Sohn, and M. Pyo, Adv. Energy Mater. 8, 1703099 (2018).

30. N. Naveen, W. B. Park, S. C. Han, S. P. Singh, Y. H. Jung, D. Ahn, K.-S. Sohn, and M. Pyo, Chem. Mater. 30, 2049 (2018).

31. J.-W. Lee, S. P. Singh, M. Kim, S. U. Hong, W. B. Park, and K.-S. Sohn, Inorg. Chem. 56, 9814 (2017).

32. Y. H. Jung, W. B. Park, M. Pyo, K.-S. Sohn, and D. Ahn, J. Mater. Chem. A 5, 8939 (2017).

33. B. D. Lee, W. B. Park, J.-W. Lee, M. Kim, M. Pyo, and K.S. Sohn Chem. Mater. 33, 782 (2021).

34. J.-W. Lee, W. B. Park, J. H. Lee, S. P. Singh, and K.-S. Sohn, Nat. Commun. 11, 1 (2020).

35. J.-W. Lee, W. B. Park, B. D. Lee, S. Kim, N. H. Goo, and K.-S. Sohn, Sci. Rep. 10, 1 (2020).

36. K.-S. Sohn, J. Chung, M.-Y. Cho, S. Timilsina, W. B. Park, M. Pyo, N. Shin, K. Sohn, and J. S. Kim, Sci. Rep. 7, 1 (2017).

37. W. B. Park, J. Chung, J. Jung, K. Sohn, S. P. Singh, M. Pyo, N. Shin, and K.-S. Sohn, IUCrJ 4, 486 (2017).

38. M. L. Agiorgousis, Y.-Y. Sun, D.-H. Choe, D. West, and S. Zhang, Adv. Theory Simul. 2, 1800173 (2019).

39. P. V. Balachandran, A. A. Emery, J. E. Gubernatis, T. Lookman, C. Wolverton, and A. Zunger, Phys. Rev. Mater. 2, 043802 (2018).

40. C. J. Bartel, C. Sutton, B. R. Goldsmith, R. Ouyang, C. B. Musgrave, L. M. Ghiringhelli, and M. Scheffler, Sci. Adv. 5, eaav0693 (2019).

41. W. G. Han, W. B. Park, S. P. Singh, M. Pyo, and K.-S. Sohn, Phys. Chem. Chem. Phys. 20, 26405 (2018).

42. S. U. Hong, S. P. Singh, M. Pyo, W. B. Park, and K.-S. Sohn, Phys. Chem. Chem. Phys. 19, 16702 (2017).

43. W. B. Park, S. U. Hong, S. P. Singh, M. Pyo, and K.-S. Sohn, ACS omega 1, 483 (2016).

44. J. P. Perdew, K. Burke, and M. Ernzerhof, Phys. Rev. Lett. 77, 3865 (1996).
45. G. Kresse and J. Hafner, Phys. Rev. B. 47, 558 (1993).

46. G. Kresse and J. Hafner, Phys. Rev. B. 49, 14251 (1994).

47. G. Kresse and J. Furthmüller, Comput. Mater. Sci. 6, 15 (1996).

48. G. Kresse and J. Furthmüller, Phys. Rev. B. 54, 11169 (1996).

49. P. E. Blöchl, Phys. Rev. B. 50, 17953 (1994).

50. G. Kresse and D. Joubert, Phys. Rev. B. 59, 1758 (1999).

51. P. S. Whitfield, N. Herron, W. E. Guise, K. Page, Y. Q. Cheng, I. Milas, and M. K. Crawford, Sci. Rep. 6, 35685 (2016).

52. P. Docampo and T. Bein, Acc. Chem. Res. 49, 339 (2016).

53. Y. Rong, L. Liu, A. Mei, X. Li, and H. Han, Adv. Energy Mater. 5, 1501066 (2015).

54. T. Leijtens, G. E. Eperon, N. K. Noel, S. N. Habisreutinger, A. Petrozza, and H. J. Snaith, Adv. Energy Mater. 5, 1500963 (2015).

55. T. A. Berhe, W.-N. Su, C.-H. Chen, C.-J. Pan, J.-H. Cheng, H.-M. Chen, M.-C. Tsai, L.-Y. Chen, A. A. Dubale, and B.J. Hwang, Energy Environ. Sci. 9, 323 (2016).

56. M. Ye, X. Hong, F. Zhang, and X. Liu, J. Mater. Chem. A 4, 6755 (2016).

57. N. H. Tiep, Z. Ku, and H. J. Fan, Adv. Energy Mater. 6, 1501420 (2016).

58. F. Brivio, J. M. Frost, J. M. Skelton, A. J. Jackson, O. J. Weber, M. T. Weller, A. R. Goñi, A. M. A. Leguy, P. R. F. Barnes, and A. Walsh, Phys. Rev. B 92, 144308 (2015).

59. B. Li, Y. Li, C. Zheng, D. Gao, and W. Huang, RSC Adv. 6, 38079 (2016).

60. Q. Wang, Q. Dong, T. Li, A. Gruverman, and J. Huang, $A d v$. Mater. 28, 6734 (2016).

61. J. H. Lee, S. P. Singh, M. Kim, M. Pyo, W. B. Park, and K.S. Sohn, Inorg. Chem. Front. 6, 3493 (2019).

62. M. Kim, W. B. Park, J.-W. Lee, J. Lee, C. H. Kim, S. P. Singh, and K.-S. Sohn, Chem. Mater. 30, 6936 (2018).

63. S. P. Singh, M. Kim, W. B. Park, J.-W. Lee, and K.-S. Sohn, Inorg. Chem. 55, 10310 (2016).

64. W. B. Park, H. Kim, H. Park, C. Yoon, and K.-S Sohn, Inorg. Chem. 55, 2534 (2016).

65. M. Kim, W. B. Park, B. Bang, C. H. Kim, and K.-S. Sohn, J. Mater. Chem. C 4, 1914 (2016).

66. M. Kim, W. B. Park, B. Bang, C. H. Kim, and K.-S. Sohn, J. Mater. Chem. C 3, 5484 (2015).

67. W. B. Park, S. P. Singh, M. Kim, and K.-S. Sohn, Inorg. Chem. 54, 1829 (2015). 